\title{
SPIN STRUCTURE FUNCTIONS: PROTON/DEUTERON MEASUREMENTS IN THE RESONANCE REGION
}

\author{
M. K. JONES FOR THE $R S S$ COLLABORATION* \\ Jefferson $L a b$ \\ 12000 Jefferson Ave. \\ Newport News, VA 23606, USA \\ E-mail: jones@jlab.org
}

The RSS experiment ran in Hall $\mathrm{C}$ at Jefferson Lab and measured the proton and deuteron beam-target asymmetries for parallel and perpendicular target fields over a $W$ range from pion threshold to $1.9 \mathrm{GeV}$ at $Q^{2} \approx 1.3 \mathrm{GeV}^{2}$. Preliminary results for the proton spin structure functions $g_{1}$ and $g_{2}$ are presented.

\section{Introduction}

This workshop has highlighted the large amount of data that is available for studying quark-hadron duality in the unpolarized structure functions and making precision tests of duality (see review ${ }^{1}$ ). With precision measurements in the resonance region, examination of duality in the $g_{1}$ and $g_{2}$ spin structure functions (SSFs) can also be done. A model independent determination of $g_{1}$ and $g_{2}$ can be done with measurement of the beamtarget asymmetry at two different orientations of the target spin. $g_{1}$ has been measured in the DIS region for a wide range of $Q^{2}$. At SLAC, $g_{2}$ and $g_{1}$ were measured in the resonance region, but with large $W$ bins. In Hall B at Jefferson Lab, precision data for the parallel beam-target asymmetries on the proton and deuteron in the DIS and resonance regions have been measured ${ }^{2,3,4}$ over a large range of moderate $Q^{2}$ and $g_{1}$ was extracted using estimates of $g_{2}$. Recent measurements ${ }^{5}$ of neutron $g_{1}$ and $g_{2}$ in the resonance region using a polarized ${ }^{3} \mathrm{He}$ target have been done at Jefferson Lab

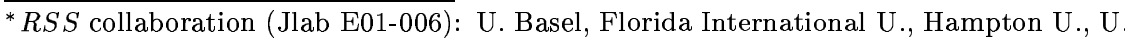
of Massachusetts, U. of Maryland, Mississippi State U., North Carolina A\&T STATE U., U. of N. C. at Wilmington, Norfolk State U., Old Dominion U., S.U. at New Orleans, U. of Tel-Aviv, Jefferson Lab, U. of Virginia, Virginia P. I. \& S.U., Yerevan Physics Institute. Spokesmen: O. A. Rondon and M. K. Jones . 
at $Q^{2}<1$ and preliminary results of another Hall A experiment ${ }^{6}$, which studied the dependence of neutron $g_{1}$ and $g_{2}$ in the resonance region for $1<Q^{2}<4 \mathrm{GeV}^{2}$, have been presented at this workshop. A lack of data for the proton $g_{2}$ in the resonance region at low $Q^{2}$ is a glaring hole in the kinematic map of SSFs. The goal of the Hall C $R S S$ experiment is to measure the $\mathrm{W}$ dependence of proton and deuteron $g_{1}$ and $g_{2}$ in the resonance region at a $Q^{2} \approx 1.3 \mathrm{GeV}^{2}$.

In the $R S S$ experiment, beam-target asymmetries were measured for proton and deuteron targets with the target spin parallel $\left(A_{\|}\right)$and perpendicular $\left(A_{\perp}\right)$ to the beam helicity. The $A_{\|}$and $A_{\perp}$ can be combined to determine the spin structure functions $g_{1}$ and $g_{2}$ or the related spin asymmetries $A_{1}$ and $A_{2}$. Duality can be studied for both $g_{1}$ and $g_{2}$ and, with precision data in small $W$ bins, local quark-hadron duality can be investigated. While $g_{2}$ does not have a simple partonic interpretation, $g_{2}$ is sensitive to higher twist.

\section{Experiment}

The RSS experiment was performed in Hall $\mathrm{C}$ at the Thomas Jefferson National Accelerator Facility (Jefferson Lab). Polarized electrons with $5.76 \mathrm{GeV} / \mathrm{c}$ momentum were scattered from polarized frozen ammonia $\left(\mathrm{NH}_{3}\right)$ and deuterated ammonia $\left(\mathrm{ND}_{3}\right)$ targets. The spin of the polar-

ized target was aligned anti-parallel and perpendicular to the beam. The scattered electrons were detected at $13.15^{\circ}$ in the High Momentum Spectrometer (HMS). Electron particle identification was done by a combination of a gas Cerenkov detector and lead-glass calorimeter. To cover the range of $W$ from $0.6 \mathrm{GeV}$ to $1.9 \mathrm{GeV}$, data was taken with the HMS at two central momentum settings of 4.73 and $4.09 \mathrm{GeV} / \mathrm{c}$. The average momentum transfer-squared, $Q^{2}$, was centered at $1.3 \pm 0.3 \mathrm{GeV}^{2}$ for $1.1<W<$ 1.9. $\mathrm{GeV}$.

The ammonia targets were polarized by dynamic nuclear polarization and operated at $1 \mathrm{~K}$ in a $5 \mathrm{~T}$ magnetic field. To maintain reasonable target polarization, the beam current was limited to $<100 \mathrm{nA}$ and was uniformly rastered over a $2 \mathrm{~cm}$ diameter circle. The target polarization, $P_{T}$, was measured continuously by the NMR technique. The average proton polarization was $70 \%$ and the average deuteron polarization was $20 \%$. The polarization of the beam, $P_{B}$, was measured in Hall $\mathrm{C}$ using the Moller polarimeter. The average $P_{B}$ was $65.6 \%$ (71\%) for perpendicular (parallel) target field. 
The measured asymmetry, $A_{m}$, is defined as $\frac{N^{+}-N^{-}}{N^{+}+N^{-}}$where $N^{+}$and $N^{-}$ are the raw counts normalized for deadtime and charge for opposite beam helicities. The asymmetry for parallel and perpendicular target field is

$$
A_{\|, \perp}=\frac{1}{C_{N} f_{R C}}\left(\frac{A_{m}}{f P_{B} P_{T}}-C_{D}\right)+A_{R C}
$$

where the measured asymmetry is normalized by $P_{T}, P_{B}$ and the dilution factor, $f$. The dilution factor is the ratio of the yield from polarized free protons to yield from all target material. The asymmetry is also corrected for radiative effects $\left(f_{R C}\right.$ and $\left.A_{R C}\right)$ and the contribution from the small nitrogen polarization $\left(C_{N}, C_{D}\right)$. The $C_{N}$ correction is $\approx 1.02$ has not been applied to the preliminary data that is being shown. The $C_{D}$ correction is for deuteron target only.

Elastic $e p$ asymmetry, $A_{e p}$, was measured when the HMS central momentum was $4.73 \mathrm{GeV} / \mathrm{c}$. The sensitivity of $A_{e p}$ to the ratio of the proton electric to magnetic form factor, $\frac{G_{E}}{G_{M}}$, depends on angle between the target spin and the momentum transfer. For parallel target field, $A_{e p}$ is insensitive to $\frac{G_{E}}{G_{M}}$ and was used to determine $P_{B} P_{T}$ to a combined relative statistical and systematic error of $1.9 \%$. For the perpendicular target field, the sensitivity of $A_{e p}$ to $\frac{G_{E}}{G_{M}}$ is large enough that $\frac{G_{E}}{G_{M}}$ can be determined from $A_{e p}$ using $P_{B} P_{T}$ determined by NMR technique and Moller polarimeter.

\section{Results}

Preliminary results for the proton spin structure functions, $g_{1}^{p}$ and $g_{2}^{p}$, from the $R S S$ experiment are plotted versus Bjorken $x_{b j}$ in Fig. 1a and Fig. 1b respectively. In Fig. 1a, previous $g_{1}^{p}$ data $^{7}$ from SLAC E143 at $Q^{2} \approx 5 \mathrm{GeV}^{2}$ and data ${ }^{2}$ from Jefferson Lab Hall B at $Q^{2} \approx 1.35 \mathrm{GeV}^{2}$ are plotted for comparison. While agreement is good for $x_{b j}>0.5$, the RSS data exhibit more structure in the $0.5>x_{b j}>0.3$ region compared to the Hall B data. But at this time, the $R S S$ data are still preliminary and radiative corrections are being finalized. The $R S S g_{1}^{p}$ are compared with calculations from three different $\mathrm{PDFs}\left(\mathrm{BSB}^{9}, \mathrm{GRSV}^{10}\right.$ and $\mathrm{AAC}^{11}$ ) which have been evolved to $Q^{2}=1.3 \mathrm{GeV}^{2}$. The GRSV and AAC PDFs have target mass corrections. The $R S S$ data oscillate around the PDF curves.

In Fig. 1b, arrows indicate the associated $W$ for the given $x_{b j}$ for the $R S S$ experiment. Here, data ${ }^{8}$ for $g_{2}^{p}$ from SLAC E155 are plotted. The SLAC data are at higher $Q^{2}$ and in the DIS region. The $R S S g_{2}^{p}$ has significant structure in the $0.5>x_{b j}>0.3$ region. 

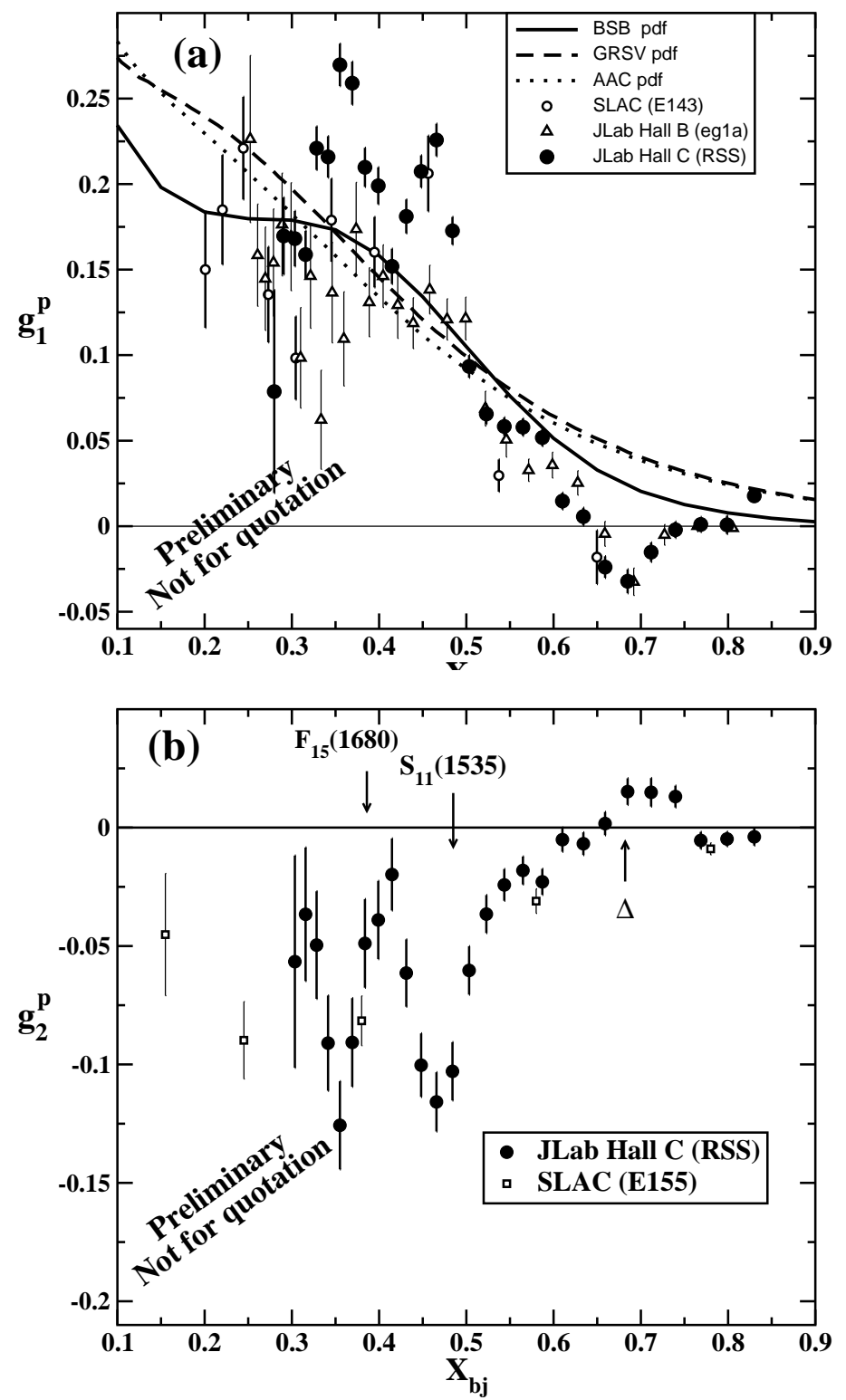

Figure 1. The proton structure functions $g_{1}^{p}$ and $g_{2}^{p}$. The curves are described in the text. 
Once the data are finalized, ratios of data to the PDFs can be made that will enable quantitative tests of duality in proton SSFs. In addition, the $R S S$ experiment measured deuteron beam-target asymmetries and these data will be combined with the proton data to extract neutron SSFs.

\section{Acknowledgments}

This work was supported in part by the U. S. Department of Energy (DOE) contract DE-AC05-84ER40150, under which the Southeastern Universities Research Association (SURA) operates the Thomas Jefferson National Accelerator Facility (Jefferson Lab).

\section{References}

1. W. Melnitchouk, R. Ent and C. Keppel, Phys. Rept. 406, 127 (2005).

2. R. Fatemi et al., Phys. Rev. Lett. 91, 222002 (2003).

3. J. Yun et al., Phys. Rev. C67, 055204 (2003).

4. Y. Prok, These proceedings.

5. M. Amarian et al., Phys. Rev. Lett. 92, 022301 (2004).

6. P. Solvignon, These proceedings.

7. K. Abe et al., Phys. Rev. D58, 112003 (1998).

8. P. Anthony et al., Phys. Lett. B533, 17 (2003).

9. C. Bourrely, J. Soffer and F. Buccella, Eur. Phys. J. C23, 487 (2002).

10. M. Glueck, E. Reya, M. Stratmann and W. Vogelsang, Phys. Rev. D63, 094005 (2001).

11. M. Hirai et al., Phys. Rev. D69, 054021 (2004). 